\title{
Exploring what motivates evaluation capacity building in early learning services: "What are the children getting out of this?"
}

\section{Elena F. Moretti}

This article describes a research project focused on evaluation capacity building and internal evaluation practice, in a small sample of early learning services in Aotearoa New Zealand. Poor evaluation practice in this context has persisted for several decades, and capacity building attempts have had limited impact. Multiple methods were used to gather data on factors and conditions that motivated successful evaluation capacity building and internal evaluation practice in five unusually high-performing early learning services.

The early learning sector context is described and discussed in relation to existing research on evaluation capacity building in organisations. This is followed by a brief overview of the research methodology for this study, with the majority of the article devoted to findings and areas for future exploration and research. Quotes from the research participants are used to illustrate their views, and the views of the wider early learning sector, on evaluation matters. 
Findings suggest that motivation is hindered by a widespread view of internal evaluation as overly demanding and minimally valuable. In addition, some features of the Aotearoa New Zealand early learning context mean that accountability factors are not effective motivators for evaluation capacity building. Early learning service staff are more motivated to engage in evaluation by factors and conditions related to their understandings of personal capability, guidance and support strategies, and the alignment of internal evaluation processes to positive children's outcomes.

The strength of agreement within the limited sample size and scope of this study, particularly considering the variation in early learning service contexts of the research participants, supports the validity of the findings. Understandings of evaluation capacity building motivators in this context will contribute to discussions related to organisation evaluation, internal evaluation, social-sector evaluation, and evaluation capacity building.

\section{Introduction}

It is well-established that high-quality internal evaluation (IE) is a key contributor to high-quality provision for children in early learning services in Aotearoa New Zealand (Education Review Office, 2010, 2013a, 2016a, 2020). To this end, licensed early learning services are required to regularly engage in evaluative processes to review and improve their quality. The purpose of IE is described by the Education Review Office (2016b) as being "undertaken to assess what is and is not working, and for whom, and then to determine what changes are needed ... IE involves asking good questions, gathering fit-for-purpose data and information, and then making sense of that information" (p. 4). (The Education Review Office is Aotearoa New Zealand's government department that evaluates and 
reports on the quality of early learning services, as well as taking an active role in promoting evaluation capacity [2018].) Over several decades, various strategies and resources have been put in place by the Ministry of Education and the Education Review Office to support the sector with their understanding and use of IE. ${ }^{1}$

Research conducted by the Education Review Office shows that poor IE practice has persisted in the sector and continues to require significant development (2009, 2013b, 2016a), indicating that capacity building attempts to date have had limited impact. IE within an Aotearoa New Zealand early learning setting should be "primarily concerned with the service's impact on children's learning and development" (Ministry of Education, 2017, p. 65). Instead, most services focus on measuring adult inputs rather than children's learning and development outcomes, consist mostly of anecdotal reflections, or use an IE framework to conduct research and development projects that have no evaluative aspect. The ongoing low quality of IE practice suggests limited motivation towards evaluation capacity building (ECB), where early learning services would have deliberately built their evaluation understandings and practices to "make quality evaluation and its uses routine" (Stockdill et al., 2002, p. 14). There appears to be an ongoing issue with IE not being valued or understood by the sector overall. Perhaps the central issue here is as described by Maloney (2019): "As evaluators, we see evaluation as a gift ... We see how evaluation can support ... better individual, social and environmental outcomes. But we also know that not everyone sees evaluation this way" (p. 1).

It should be considered that the early learning context differs in many ways from other sorts of organisations, particularly in terms of

1 The research described was partially funded by the Education Review Office. The research described was submitted to Massey University as a research project, 2020. 
accountability measures, as will be discussed in the next section. It seemed likely that the unique qualities of the early learning context may have unique impacts on motivation to engage in ECB. Preskill and Boyle (2008) write about the "'trigger' or motivating reason" that prompts an organisation's ECB (p. 446). The purpose of this research was to understand these "motivating reason[s]" within a sample of atypical early learning services that are excelling at ECB and IE. This study draws on multiple methods to discover motivators and conditions that contributed to their successful ECB and robust IE practice, with the potential to inform ECB strategies better suited to the special characteristics of the sector.

\section{Considering the early learning sector context}

Unlike many organisations, early learning services do not have accountability functions related to any obvious or easily measurable outputs. Research literature on ECB and IE shows that accountability around outputs, or outcomes, can be an effective motivator when an organisation is motivated to engage in ECB because of underperformance or needing to meet requirements (Compton et al., 2001; Preskill \& Boyle, 2008). While educational organisations do not measure their performance by units produced or profits made, schools and universities can still have outcomes compared at a glance, through normed assessments, national-qualification programmes, and the like. It makes sense that, in the school context, "a shift in emphasis to schools demonstrating accountability for outcomes has raised expectations in relation to school internal/ self-evaluation" (Education Review Office, 2011, p. 4). Assessment of learning outcomes in early learning services, however, is usually non-standardised, relational, and highly individualised, building upon a child's own knowledge, skills, dispositions, and sociocultural context rather than easily comparable indicators (Ministry of 
Education, 2017). This could make it particularly difficult for early learning service staff to have any sense of "underperformance". The sector's limited IE capacity (Education Review Office, 2009, 2013b, 2016a) also means they are less equipped to discover underperformance themselves, through IE practices.

Funding allocation linked to performance is also a catalyst for ECB (Compton et al., 2001; Stevenson et al., 2002). Chapman and Sammons (2013), for example, link IE in English schools to the provision of "proof to key stakeholders ... that they are providing value for money" (p. 11). It may be relevant that early learning services in Aotearoa New Zealand are funded upon meeting minimum compliance standards, with no relationship to quality levels beyond those standards. In terms of IE, early learning services are legislatively required to conduct some form of evaluation, but the robustness of those evaluation processes is not mandated. There are no funding rewards or other incentives for improving evaluation practice, nor are their significant consequences if evaluation is poor.

From experience working with and within early learning services, it seemed likely that ECB engagement would be hindered by common issues observed in the sector, such as variability of teacher-training programmes; confusion and mixed messages about what IE looks like and is for; and varying accessibility, and variability in quality, of professional-learning opportunities. In addition, working conditions can be challenging in terms of time and resources. Carr et al. (2000) outline the "reality" of the sector:

Demands from families, requirements from management, typical levels of turnover in a low-paid profession ... staff picking up children's illnesses, staff meetings outside working hours, large group sizes: all these are part of the typical life of an early childhood educator. They mean that documented evaluation procedures will often be delayed. (p. 60) 
Given this context, time and resource constraints were likely to have a hindering effect on motivation for ECB. It would be useful to explore how IE processes were prioritised, or not, when working with these limitations.

Research on IE and ECB, including within education contexts, points to attitudes and emotional investment being foundationally important (Lai \& Kushner, 2013; McNamara et al., 2011; Patton, 2008; Schildkamp \& Visscher, 2009; Schulha et al., 2016). Preskill and Boyle (2008) collate a number of researchers' findings regarding ECB motivators, including the accountability factors discussed above. They posit that these motivators are likely to be "concomitant with ... assumptions that evaluation is a 'good' thing to do, that evaluation can contribute to effective decision making, and that evaluation adds value to the organisation" (p. 446). It may be the case that the sector does not consider IE to be valuable, resulting in a lack of motivation to engage with the process. Why, after all, would kaiako (a collective reference for teachers, staff, and parents in early learning settings) envisage any value added from evaluation, if accountability is low, if underperformance has little consequence? How can they know the effectiveness - or ineffectiveness-of their decision making without clear output measures? These questions warranted further exploration in this research.

Cousins et al. (2014) have noted that, while individuals and organisations may know what good evaluation practice looks like, building their ability to do and use evaluation-ECB-is another matter. The literature tends to focus on the how of ECB. This research takes a step further back, to explore: Why would we want to? To successfully promote IE practice, and its benefits for children, a better understanding is needed of what factors and conditions successfully motivate ECB: What would motivate the sector? 


\section{Research methodology}

Using multiple methods, this research explored the motivators and conditions that contributed to effective ECB within high-performing early learning services. The focus was not on how, but why, these services built and maintained high-quality IE practices. This meant gaining understandings of views, values, and other personal factors, in hopes of finding some commonality among services that had successfully built their IE practice. An analysis around the strength of IE and ECB practice, as well as regional and service-type representation, was the basis of the final selection of a purposive sample of five services.

Selection of the services was based on a three-stage process, drawing on the Education Review Office's reports and evidence files. At the first stage of the selection process, online reports were analysed and coded for indications of strong IE and ECB practices. Twentyfive Education Review Office review evidence files were accessed, and IE samples and meeting notes were examined and scored against quality indicators related to ECB and high-quality IE (Education Review Office, 2013a).

A challenge when studying the early learning sector is that the almost 4,000 mainstream early learning services in Aotearoa New Zealand are far from homogeneous. There is enormous variation, including services' structures and qualification demands, accessibility of professional development opportunities across regions and service types, and structures and effectiveness of numerous governing bodies. While the sector differs markedly from other sorts of organisations - even educational ones-it also contains much difference within itself. This had ramifications for the selection process, in order to gain as diverse a sample as possible. Participating services included representation from a large corporate organisation, a large non-profit 
teacher-led organisation, a large non-profit parent-led organisation, a small privately owned service, and a small community-based nonprofit service. Services were located in large and mid-sized cities, small towns, and rural contexts across Aotearoa New Zealand.

Initial contact was made via telephone and email. Participants in the study had responsibility for building the evaluation capacity of the team of kaiako who produced the IE samples on file at the Education Review Office. In one service, two leaders who both had ECB leadership roles participated in the research.

The research participants and their early learning services were not previously known to the author. Appropriate ethical procedures were undertaken throughout the process, including approval from the Massey University Human Ethics Committee and opportunities for participants to review transcripts and analyses.

Initial data were gathered through questionnaires. These included basic information questions as well as attitude scales; descriptive prompts about ECB motivations and the effectiveness of these; and a mind-map exercise, which asked participants to consider the difference between kaiako who do and do not value IE. Thematic analysis (Braun \& Clarke, 2006) of questionnaires identified topics for discussion in semistructured face-to-face interviews.

Ongoing memo-keeping contributed to unitisation of the data from both the questionnaires and interview transcripts, broadly based on the themes of leadership, workplace culture, "type" of people, the role of overseeing bodies and external evaluators, values, strategies, misperceptions of IE, and professional-learning experiences. Knowing there were patterns to explore in these areas, the unitised data were revisited to establish more specific analytic coding units. The coded data could then be grouped into categories, broader concepts, and then inform conclusions. 


\section{Findings and discussion}

Thematic analysis of the questionnaire and interview data led to six overall findings. These findings were either expressed by five of six participants, or unanimous. In this section, findings are presented with illustrative quotes from both the questionnaires and interviews. Discussion in the context of ECB literature and considerations for future research follow in the next section.

It should be emphasised that there was surprising strength of agreement between the motivating factors and conditions of those working in very different contexts. The early learning service settings of the participants, with their differing hours of work, pay and benefits, team makeup and qualification levels, parent or volunteer involvement, guiding philosophies, professional-learning opportunities and barriers, and leadership structures could have resulted in vastly different factors and conditions identified between settings. The unanticipated strength of agreement despite differences in context made for stronger conclusions.

\section{A strong sense of service and improvement orientation moti- vated ECB}

Participants demonstrated self-challenging attitudes, which they linked to a strong sense of professional and social responsibility. All stated with certainty that outside influences, such as funding, pressure from governing organisations, or external evaluations, were not the catalyst for their own ECB. Instead, frustration at having an unsolved area of practice motivated action. Participants described feelings of "stubborn[ness]" or having a "fascination with things I can't understand". "When I wasn't happy or satisfied with [professional development course on IE], I wanted to know more. I had to dig." "If we just wait, it's not going to happen. It's about being proactive." "Basically, let's conquer this." 
A commitment to the teaching profession and a sense of service were strong motivating themes. Participants wanted to support their teaching teams and benefit children and families. In questionnaires, participants wrote of their "desire to help", "commitment", and "not wanting to let [colleagues] down". They expressed the same sense of obligation and service in interviews; participants took their leadership role seriously and considered their own ECB a duty to their learning community. "What are the teachers getting out of this, what are the children getting out of this? So I decided to take another look at it."

There's my personal interest in education which motivated me to want to take the next step and find out about this thing... And then there's my own personal ownership of [parent-led organisation], because I want my [service] to be great, and to do all the things that it's supposed to do. I feel ownership over that, so that means that I feel like it's my responsibility to understand this.

\section{Participants identified as naturally orderly and systematic, which supported their motivation to engage in ECB and IE processes}

Almost all participants identified as certain "type[s] of people" who find IE satisfying. They aligned their IE capability with a natural inclination to compartmentalise and prioritise. "I'm a systems person." "I have that analytical step thing. I love doing pictures and putting things in steps and lists. We're going to do this and this and this."

While there was no noticeable commonality in participants' length of experience as kaiako or in early learning leadership positions, a pattern was evident in work experiences prior to early learning. These related to organisation of data and resources, such as management, administration, banking, science, economics, and mathematics. Participants affirmed that this could have contributed 
to their IE capability and motivation. "I knew that because of my educational background, that I was capable of understanding. I was confident." "I had to be super organised ... but I'm like that anyway."

Within interviews, participants consistently related their IE and ECB competence to being a certain "type" of person, and related the early learning sector's lack of motivation and interest in IE to many kaiako not seeing themselves as, for example, "academic". Participants described how those they worked with would insist they were not IE "type[s ]" of people: "not 'form' sorts of people”.

IE seems like a really academic process, often, from the outside. So there are people that will just immediately discount themselves from that, and they go, 'I'm not that kind of person, I won't be able to do that.'

\section{Demotivating misunderstandings about IE were widespread and difficult to shift}

Participants all agreed that kaiako — those who are not that "kind of person"-were put off by their impressions of IE. It was considered to be a large, difficult, time-consuming compliance exercise, and was described by participants as "too big ... too complex", "unattainable ... burdensome", "daunting", and "boring". They attributed this way of seeing IE as being a significant demotivator in the sector.

I think it's the big notion about IE that puts people off. Too academic, and it needs lots of research, needs lots of time, and lots of writing ... That it's time-consuming, that it's a research thing, that there should be lots of surveys, lots of data-gathering ... The preconceived notion of what it is, is what stops people from engaging in an authentic way ... it doesn't have to be flash.

Misunderstandings around IE were exacerbated by a problematic professional-learning landscape. Participants had found clear, useful resources and good-quality professional guidance difficult or 
impossible to locate. "Teachers want examples. To see what it looks like in practice. What it actually looks like." They described having to "piece together" their understandings of the IE process from paid providers; mentors; professional cluster groups; resources sourced from the Ministry of Education, the Education Review Office or the service's governing organisation; and out-of-sector internationally-based research and tools. Participants agreed that increased availability of quality professional-learning opportunities would be beneficial for the sector.

What was really hard at the start was not having a picture in mind of an exemplar. I basically had never seen someone else's, or any other centre's IE. It felt like I was working in the dark, as to what it was that we were creating.

Participants denounced "tick-box" evaluation processes that "waste time", "just chucking evidence in or filling up this box". A variation of the term "tick-box" was used by almost all participants. Some described being uninspired by professional-learning courses that seemed to promote this approach. They linked this kind of IE practice with the sector's low motivation to engage with IE, as well as their own impressions of IE prior to their ECB.

You were doing it to tick a box. Whereas [now], you do it because you want to evaluate to see how well you've done it ... because the workload is big, teachers don't want to waste time on something that they can't see a use for.

Participants expressed frustration and disappointment about ineffective professional-learning providers and the limited support available from more credible sources. "Publications can be useful but need to come with more help to understand how to put them into practice in an effective way." "We come in here and we are running, all day. Because we're motivated, we're busy. If it's just given to me in a pamphlet, it's like-I don't have time for this." 


\section{Leaders used targeted strategies to successfully boost the ECB motivation of their teams}

Participants found that by addressing their teams' demotivating sense of low capability, and their belief that IE is "too hard" or "tickbox", kaiako could become motivated to engage in ECB and the IE process. Purposeful conversations and coaching were focused on highlighting the skills kaiako already employ, and linking these to evaluation competencies. Teams were encouraged to see themselves as natural evaluators. "Teachers would say, 'I don't know how to do evaluation.' I couldn't believe it. In conversations about it though, they could say, 'Oh, I'm doing that." "For them to be able to embrace the process, it's for them first to understand that this is something you are regularly doing. Even in your staff meetings, it's naturally occurring."

To grow evaluation capability, participants strongly affirmed the use of simplified, stepped IE tools, which build capability and understanding over time through participation. Strong oversight and consistent messages about keeping a narrowed focus was also important. "Teachers are very good at making things too big, too complex." Small, achievable steps supported teachers to experience success, which participants report has had multiple benefits including keeping momentum, building confidence, and correcting the "too big" perception of IE. "I think breaking it apart really helps the new teachers ... By the middle of it they're more confident." "The workload's not as big then, it's like bang, done. How easy was that?" Constantly revisiting and refining their tools, alongside their teams of kaiako, had resulted in tailored processes specific to their early learning services. "We wanted to have less admin, more fun-so that motivated us to have more efficient ways of doing things."

Participants motivated teams with clear messages about valuing IE. For example, when asked about the successful ECB of her team, 
one participant reflected: “To be honest I think it's because I believe in it. And I kind of drive the team. I think you have to have that one person to lead it." Some leaders credited having an unusually long-serving team of kaiako as an enabler of their services' IE success. Within interviews, it was discussed whether participants believed their services had the capacity to continue IE practices without their leadership. Participants expressed hesitation. They had some, but not strong, confidence. For example, one participant responded, "I hope so. I really hope so. It's been six years, it's like ... come on guys [laughs]."

\section{Sustained work to establish an improvement-focused work- place culture contributed to ECB}

Participants described the value of keeping momentum in ECB, working closely alongside kaiako and having regular conversations that reiterate IE's purpose and process. "Explaining it to them-not bombarding them-I keep it this [gestures small, narrow]." "Walking side-by-side, to keep up ... It's not a race." "It's just chipping away." Collaborative approaches worked well, such as whole-team planning sessions and strengths-based delegation of tasks. Ensuring IE was a regular meeting-agenda item, and prioritised over more minor discussion topics, was an effective strategy.

If it gets too strung out ... they'll get very fired up about the first part, with ideas, and then it'll be missed off a couple of monthly meetings, and then it goes off the boil and everyone forgets about it.

Building a team mindset of valuing mistakes, risk-taking, and critique was important. Participants had embedded practices that encourage critical thinking and an improvement orientation within a culture of professional trust. This featured, for example, distributed leadership opportunities, regular team discussion and debate, peer observation practices, and peer or 360-degree feedback mechanisms 
in performance appraisals. Participants and their teams valued trial and error: "We're only human." "We're not perfect." "It's okay to try something and it didn't give you exactly what you wanted." They were not defensive about their practice and welcomed new learning.

[My teaching team] can try this, and it doesn't matter if it doesn't work. We can try something else ... [My regional organisation] shares our mahi, our successes, our failures. We're on the same waka ... [My regional manager and I] have a great relationship. I love it when she comes in. I keep saying to her, 'Tell me, because I don't know what I don't know. Feed me information.'

The value of an improvement orientation came through strongly in the questionnaires' mind-map exercise, when participants described differences between kaiako who do and do not engage with IE. "Practice becomes stagnant and routine, rather than purposeful, intentional and visionary." "Has open mindset, likes change", as opposed to, "Closed mindset, doesn't like change." In interviews, participants warned against ECB in an early learning service that had not taken steps to build a critical and learning-focused climate. It is crucial for IE and ECB that the team culture is "non-threatening" and "non-judgemental". "Trust" was consistently cited as a key element of an IE-ready service. Without these conditions in place, IE would be met with fear and avoidance. "IE has to feel like it's not about pointing fingers and saying, 'You're doing something wrong'."

\section{IE experiences that lead to positive impacts for children strongly motivated further ECB}

Participants found that when the topic of their IE was one of particular personal investment for their kaiako, there was a noticeable increase in the team's motivation, engagement, and confidence. "Teachers enjoy the research when it's something they really want to know, that they really want to improve." They found that focusing IE 
on positive children's outcomes would most reliably generate enthusiasm for the process: "What are we going to find out, that we don't know now, in order to be able to achieve that vision?" Over time, kaiako would become increasingly engaged with the documentation and data-measurement aspects of IE, as a way to confirm benefits for children.

We always go back to the outcomes for children-have we made

a difference? ... Is this really the outcome we wanted? How many children got improvements from us making that change?

Participants found that kaiako were more motivated to grow their evaluative practice after participating in a successful IE process. One participant, for example, wrote, "I believe that once kaiako see that IE processes intertwine beautifully with their teaching practice, they will be more driven." This finding aligns with the participants' difficulty accessing guidance and exemplars: kaiako had little opportunity to see the value of IE until they experienced it themselves.

When we developed that knowledge about how IE should actually be, that's when we actually realised the value that an authentic IE can actually bring to us as practitioners, and of course to our children.

You want teachers to be invested in it, they've got to believe in why they're doing it. And the whole point is, what is going to be the end result for the children? If we're not going to get those improvements, this is a waste of time and energy.

\section{Discussion and future research}

Within the Aotearoa New Zealand early learning sector, it appears that IE has a poor reputation, viewed as overly technical, beyond the current capability of many kaiako, and of little value and relevance to teaching and learning. Revisiting Preskill and Boyle's (2008) 
statement, that organisational ECB motivators are most effective in combination with "assumptions that evaluation is a 'good' thing to do" (p. 446), it seems that early learning sector kaiako do not hold this assumption. There is some cause for optimism in this finding, in terms of ECB, as ECB motivation has been found to increase when kaiako have opportunities to see what IE "actually is" - as one participant put it - as well as what it is not, and how it can benefit children.

Being motivated by social and emotional factors is fitting to the Aotearoa New Zealand early learning sector, considering its emphasis on relational teaching, sociocultural curriculum framework, and individualised assessment approaches. Patton (2010) writes that, "for vision-and-values-driven social innovators the highest form of accountability is internal. Are we walking the talk? Are we being true to our vision? ... These become internalised questions, asked ferociously, continuously, because they want to know" (p. 13). Participants found that when the process could be aligned to impacts for children - what kaiako "want to know"-IE increased in value, leading to an increase in motivation and engagement in ECB. Gibbs et al.'s (2002) research on ECB motivation within community-based health organisations had similar findings: the depth of staff's emotional investment in the main work of the organisation could be harnessed to increase their appreciation of evaluation processes.

Some scepticism from kaiako about the usefulness of IE makes sense, if there is little evidence of its value available: participants found that useful exemplars, resources, and professional guidance were difficult or impossible to locate. It may be that widespread misunderstandings about IE is related to ineffective professional-learning provision and sector networks; further research could explore this. According to participants, a lack of useful exemplars and guidance led to misunderstandings about what IE processes involve, and it was only after these misunderstandings were corrected that motivation 
increased. It is unsurprising that IE, if viewed as pointless or overly demanding busywork, would not generate enthusiasm. If this is a widely held view of what IE looks like, or should be, it should be possible to correct this misunderstanding. This could be an area for future exploration and impact monitoring. Appropriate ECB strategies could be developed, grounded in the understanding that kaiako can be motivated to grow their practice if they are shown they are capable of IE and given reliable, relevant, and ongoing guidance. Tools and support would need to be clearly linked to teaching practice and children's learning outcomes, and tailored to the realities of a busy teaching role.

Clear, understandable processes are crucial to ECB (Department for Education and Skills, 2004; Preskill \& Boyle, 2008). It could be beneficial to explore how the time and technical demands of IE could be minimised, as these have been shown to have a negative impact on ECB motivation. Further research around the development of easing strategies and differentiated IE tools (for example, beginner-to-expert-levelled templates and guidance) could prove useful. Narratives and exemplars that focus on aspects of teaching practice that have high emotional impact for kaiako, and clearly demonstrate the benefits of IE for children, are likely to be most impactful.

According to participants, one's self-assessment in terms of their evaluation capability is a motivator for some, and a barrier for others. Successful ECB appears to rely on a purposeful, guided shift to see oneself as the "type" of person that is suited to, and capable of, IE. Gauging the accuracy of this finding with a larger cross-sector sample would be useful, as the scope of this research, which involved those in IE-leadership positions only, meant that findings about all levels of kaiako were not gained from all levels of kaiako. It could then be useful to test whether that negative self-view can be shifted with targeted support. If ECB motivation is closely related to identity and "type" of 
person, how might professional-learning support in this area better attract, and resonate with, a wider range of "sorts of people"?

If individuals' experience organising and working with data is linked to their IE capability, the implications for the wider sector should be considered. Information could be gathered around the pre-teaching experiences of those in leadership and kaiako positions. This could give a better understanding of the needs of the sector in terms of building experience in data organisation and critical thinking. Deliberate strategies to build some beginner evaluation skills may be useful, perhaps by trialling their inclusion into teacher training or certification processes. Patterns could also be noted and addressed; for example, whether experience and capability differ between age groups, relative to schooling experiences or in relation to location or other contextual factors. An evaluation capacity needs-assessment tool, such as that developed by El Hassar et al. (2020) could be useful in this work.

While participants had some similarity in their pre-teaching experiences, this study did not reveal any significant patterns around their number of years teaching, service type, location, or other sociocultural factors. A larger sample (including kaupapa Māori and Pasifika early learning settings) may reveal key similarities or differences across demographic aspects, cultural context factors, or between leaders and kaiako. This could have implications in terms of motivating factors and conditions, and be useful in decision making about targeted or differentiated strategies.

Three of the more predictable findings in this research were that ECB was effectively motivated by: leaders who understood and valued evaluation; consistent modelling and support; and an established trusting team culture. These elements are well represented in existing ECB literature (e.g., Chapman \& Sammons, 2013; Compton et al., 2002; Cousins \& Bourgeois, 2014; KujiShikatani et al., 2015; 
Rogers \& Gullickson, 2018). There is difficulty, however, in relating these findings to ECB strategies for the early learning sector, with its "reality" of high staff turnover, as noted by Carr et al. (2000, p. 60). This turnover is illustrated even within the small sample size of this study: of the six participants, four had changed their positions in the last 2 years. If successful ECB necessitates consistent and trusting relationships, ongoing knowledge-building strategies, and leadership that is competent in IE processes, what do these elements mean for services with revolving staff and leaders? This is particularly problematic when considering the evident scarcity of early learning kaiako who understand and value the IE process. Research into the effects, challenges, and opportunities of ever-changing teams in relation to ECB and IE would be of value. A better understanding of this key characteristic of the early learning sector could inform responsive strategies to build and maintain IE capacity in the sector, as well as adding to the growing body of research into organisational ECB.

\section{Conclusion}

This study provides an evidential basis for key motivating factors and conditions for ECB and IE, within the Aotearoa New Zealand early learning context. These relate to alignment of the process to positive children's outcomes, understandings of personal capability, and appropriate guidance and support strategies. The strength of agreement between participants from diverse early learning contexts, as well as the congruence between their experiences and IE and ECB research literature more generally, as well as the pattern of low IE quality in the early learning sector, support the conclusions of this study.

Findings indicate that the early learning sector does not view IE processes as valuable or worthwhile: the "necessary condition" of evaluation valuing (Patton, 2012, p. 15) is not in place. Pervasive 
misunderstandings about the purpose and demands of IE are demotivating for leaders and kaiako. Increased valuing of evaluation can be fostered with exposure to IE processes that are understandable, meaningful, emotionally relevant, and positively impactful in terms of children's outcomes.

Evaluation research has posited many sets of conditions and factors that contribute to successful ECB (for example, Compton et al., 2002; Cousins \& Bourgeois, 2014;) and IE (for example, Education Review Office, 2013a, 2020; Schildkamp \& Visscher, 2009; Vanhoof \& Van Petegem, 2013). Broadly, these sets include some form of knowledgeable and inspiring leadership, an improvement-oriented and trusting workplace culture, good professional guidance, and clear links between evaluation processes and other valued areas of the organisation's work. The findings of this study indicate that these conditions and factors also support ECB in early learning services. However, the early learning sector appears to be less motivated to grow its IE practice for external accountability reasons, such as performance measures and external evaluation, compared to other organisations. Instead, ECB is driven by personally held understandings of capability, a desire to critique and improve practice, and a strong drive to positively impact children.

To effectively motivate the early learning sector to develop robust IE practice, ECB support should be clear, useful, and easily accessible. Most importantly, kaiako must be convinced that IE has value: that it will benefit children.

\section{Acknowledgements}

The author would like to acknowledge the generosity of the participant kaiako in sharing their experiences, as well as Robin Peace, Sandra Collins, Ro Parsons and Steve Tracey for professional and technical support. 


\section{References}

Braun, V., \& Clarke, V. (2006). Using thematic analysis in psychology. Qualitative Research in Psychology, 3(2), 77-101. https://doi. org/10.1191/1478088706qp063oa

Carr, M., May, H., \& Podmore, V. (2000). Learning and teaching stories: Action research on evaluation in early childhood. Final report to the Ministry of Education. New Zealand Council for Educational Research.

Chapman, C., \& Sammons, P. (2013). School self-evaluation for improvement: What works and why? Centre for British Teachers Education Trust.

Compton, D., Baizerman, M., Preskill, H., Rieker, P., \& Miner, K. (2001). Developing evaluation capacity while improving evaluation training in public health: The American Cancer Society's Collaborative Evaluation Fellows Project. Evaluation and Program Planning, 24(1), 33-40. https:// doi.org/10.1016/S0149-7189(00)00045-8

Compton, D., Baizerman, M., \& Stockdill, S. (Eds.). (2002). Special issue: The art, craft and science of evaluation capacity building. New Directions for Evaluation, 93(Spring). https://doi.org/10.1002/ev.38

Cousins, J. B., \& Bourgeois, I. (Eds.). (2014). Organizational capacity to do and use evaluation. New Directions for Evaluation, 141. https://doi. org/10.1002/ev.2014.2014.issue-141

Cousins, J. B., Bourgeois, I., \& Associates. (2014). Multiple case study methods and findings. In J. B. Cousins \& I. Bourgeois (Eds.), Organizational capacity to do and use evaluation. New Directions for Evaluation, 141, 25-99. https://doi.org/10.1002/ev.20077

Department for Education and Skills. (2004). A new relationship with schools: Improving performance through school self-evaluation. Author.

Education Review Office. (2009). Implementing self review in early childhood services. Author.

Education Review Office. (2010). Quality in early childhood services. Author. https:/www.ero.govt.nz/publications/quality-in-early-childhood-services/ Education Review Office. (2011). External and internal school evaluation: 
A narrative review of the literature of the Evaluation indicators for school reviews (2001) and the development of a self review resource for schools, Effective self review [unpublished]. Author.

Education Review Office. (2013a). He pou tātaki: How ERO reviews early childhood education services. Author.

Education Review Office. (2013b). Priorities for children's learning in early childhood services: Good practice.

Author. https://www.ero.govt.nz/ publications/ priorities-for-childrens-learning-in-early-childhood-services-good-practice/

Education Review Office. (2016a). Early learning curriculum: What's important and what works. Author.

Education Review Office. (2016b). Effective internal evaluation for improvement. Author.

Education Review Office. (2018). External evaluation in Aotearoa New Zealand: Principles of practice. Author.

Education Review Office. (2020). Te ara poutama - Indicators of quality for early childhood education: What matters most. Author.

El Hassar, B., Poth, C., Gokiert, R., \& Bulut, O. (2020). Toward an evidence-based approach to building evaluation capacity. Canadian Journal of Program Evaluation / La Revue canadienne d'évaluation de programme. https://doi.org/10.3138/cjpe.69191

Gibbs, D., Napp, D., Jolly, D., Westover, B., \& Uhl, G. (2002). Increasing evaluation capacity within community-based HIV prevention programs. Evaluation and Program Planning, 25(3), 261-269. https://doi.org/10.1016/ S0149-7189(02)00020-4

KujiShikatani, K., Gallagher, M. J., Franz, R., \& Börner, M. (2015). Leadership's role in building the education sector's capacity to use evaluative thinking. In M. Q. Patton, K. McKegg, \& N. Wehipeihana (Eds.), Developmental evaluation exemplars: Principles in practice (pp. 252-270). Guilford Press. 
Lai, M. K., \& Kushner, S. (2013). A developmental and negotiated approach to school self-evaluation. Emerald. https://doi.org/10.1108/ S1474-7863(2013)0000014022

Maloney, J. (2019, January 30). What does it mean to 'un-box' evaluation? [Blog post]. Better evaluation. https://betterevaluation.org/blog/ what-does-it-mean-un-box-Evaluation

McNamara, G., O’Hara, J., Lisi, P. L., \& Davidsdottir, S. (2011). Operationalising self-evaluation in schools: Experiences from Ireland and Iceland. Irish Educational Studies, 30(1), 63-82. https://doi.org/10.1080/0 3323315.2011 .535977

Ministry of Education Te Tāhuhu o te Mātauranga. (2017). Te whäriki-He whäriki mätauranga mō ngà mokopuna o Aotearoa: Early childhood curriculum. Author. https:/tewhariki.tki.org.nz/en/ early-childhood-curriculum/

Patton, M. Q. (2008). Utilization-focused evaluation (4th ed.). Sage.

Patton, M. Q. (2010). Developmental evaluation: Applying complexity concepts to enhance innovation and use. Guilford Press.

Patton, M. Q. (2012). Essentials of utilization-focused evaluation. Sage.

Preskill, H., \& Boyle, S. (2008). A multidisciplinary model of evaluation capacity building. American Journal of Evaluation, 29(4), 443-459. https:// doi.org/10.1177/1098214008324182

Rogers, A., \& Gullickson, A. (2018). Evaluation champions: A literature review. Journal of MultiDisciplinary Evaluation, 14(30), 46-63.

Schildkamp, K., \& Visscher, A. (2009). Factors influencing the utilisation of a school self-evaluation instrument. Studies in Educational Evaluation, 35(4), 150-159. https://doi.org/10.1016/j.stueduc.2009.12.001

Schulha, L., Whitmore, E., Gilbert, N., \& al Hudib, H. (2016). Introducing evidence-based principles to guide collaborative approaches to evaluation: Results of an empirical process. American Journal of Evaluation, 37(2), 193-215. https://doi.org/10.1177/1098214015615230 
Stevenson, J. F., Florin, P., Mills, D. S., \& Andrade, M. (2002). Building evaluation capacity in human service organizations: A case study. Evaluation and Program Planning, 25(3), 233-243. https://doi. org/10.1016/S0149-7189(02)00018-6

Stockdill, S. H., Baizerman, M., \& Compton, D. W. (2002). Toward a definition of the ECB process: A conversation with the ECB literature. In M. Baizerman, D. W. Compton, \& S. H. Stockdill (Eds.), The art, craft, and science of evaluation capacity building. New Directions for Evaluation, 93, 7-25. https://doi.org/10.1002/ev.39

Vanhoof, J., \& Van Petegem, P. (2013). Judging and explaining the quality of school self-evaluations: Indicators and findings on meta-evaluation from a Flemish perspective. In M. Lai \& S. Kushner (Eds.), A developmental and negotiated approach to school self-evaluation (pp. 275-291). Emerald. https://doi.org/10.1108/S1474-7863(2013)0000014016

\section{The author}

Elena F. Moretti: Education Review Office, Wellington,

New Zealand

Email: Elena.Moretti@ero.govt.nz

Elena.F.Moretti@gmail.com 